\title{
Actinomyces odontolyticus isolated from the female genital tract
}

\author{
RG MITCHELL, MR CROW
}

From the Department of Microbiology, John Radcliffe Hospital, Oxford

SUMMARY Actinomyces odontolyticus was isolated from genital tract specimens from $4 \cdot 8 \%$ of 561 women fitted with intrauterine contraceptive devices and from $4 \%$ of 101 women with pelvic inflammatory disease and $1.8 \%$ of 525 women without pelvic inflammatory disease who were not known to be intrauterine contraceptive device wearers. The strains were isolated by prolonged anaerobic incubation of blood agar, with or without added $5 \%$ metronidazole or $1 \%$ neomycin. $\boldsymbol{A}$ odontolyticus has not been previously reported in cervico-vaginal specimens, and possible reasons for this are discussed.

Actinomyces odontolyticus was first described in 1958 by Batty, ${ }^{1}$ who isolated strains from deep carious dentine and saliva. On blood agar, colonies initially resembled viridans streptococci, but after three or four days' incubation they developed a characteristic dark reddish brown pigment. The strains grew equally well under aerobic or anaerobic conditions. Isolation and identification of the organism were considered to be "exceedingly difficult."

$A$ odontolyticus has only rarely been recovered from body sites other than the oral cavity, although transient bacteraemia after dental manipulation may occur. ${ }^{2}$ Disseminated lesions due to $A$ odontolyticus have occasionally been reported, including granulomatous lesions of the chest wall, ${ }^{3}$ actinomycosis of the malar region, ${ }^{4}$ pulmonary abscess, ${ }^{5}$ and pleural empyema. ${ }^{67} \mathrm{~A}$ odontolyticus was repeatedly isolated together with Fusobacterium necrophorum from the blood of a patient who developed a septicaemic illness with multisystem involvement attributed primarily to the fusobacterium. ${ }^{8}$ Mouse inoculation experiments suggested that both organisms administered together showed enhanced virulence.

In all the above cases, the source of the organism, though not established, was probably the periodontal region or oropharynx.

In this laboratory we have isolated $A$ odontolyticus from numerous cervico-vaginal specimens receiving prolonged anaerobic culture for the isolation of actinomyces, which is now widely implicated in genital infections associated with intrauterine

Accepted for publication 21 August 1984 contraceptive devices (IUCDs)..$^{9}$ These isolates and their possible importance are reported here as we are unaware of previous accounts of this organism in the female genital tract.

\section{Material and methods}

Initially, $A$ odontolyticus was isolated sporadically from seven women wearing IUCDs but disregarded. Two years later a systematic investigation was undertaken, and over a period of 14 months specimens were obtained from 561 patients fitted with IUCDs. These included high vaginal swabs, endocervical swabs, and IUCDs themselves when removal was clinically indicated. No special transport swabs or media were used, and specimens were cultured on blood agar plates, incubated aerobically and anaerobically with additional $\mathrm{CO}_{2}$, as well as in cooked meat medium. Anaerobic culture was also carried out on horse blood agar plates containing $1 \%$ neomycin sulphate, which were incubated for five days. For isolation of actinomyces, specimens were inoculated on to blood agar plates containing $5 \%$ metronidazole for the selective inhibition of strict anaerobes. These were incubated anaerobically and examined after five and 10 days' incubation at $37^{\circ} \mathrm{C}$. Both the neomycin and metronidazole plates were examined under a low power binocular microscope for typical colonies of $\boldsymbol{A}$ israelii (" molar tooth") or dark brownish red colonies characteristic of $\boldsymbol{A}$ odontolyticus. Colonies selected for further investigation were subcultured on to blood agar plates, which were incubated anaerobically.

Cervico-vaginal specimens were obtained from a 
further group of 101 patients not stated to be IUCD wearers but with suspected pelvic inflammatory disease, which warranted prolonged anaerobic culture of specimens. These were cultured by standard methods given above and inoculated on to neomycin agar plates, which were incubated anaerobically for five days.

Finally, a survey was carried out of the incidence of $A$ odontolyticus in 525 vaginal swabs from patients without pelvic inflammatory disease and not stated to be IUCD wearers. Most were submitted with a diagnosis of vaginal discharge. For this purpose the routine anaerobic blood agar plate was retained and reincubated anaerobically for up to $\mathbf{1 0}$ days.

Colonies selected as likely $\boldsymbol{A}$ odontolyticus on appearances were verified as Gram positive bacteria, and submitted to the following physiological and biochemical tests:

1 Atmospheric requirements for optimal growth on blood agar.

2 Pigmentation on blood agar, Isosensitest agar containing lysed blood, and Columbia agar.

3 Twenty eight strains were identified by the Minitek Anaerobe II System (Becton Dickinson Limited), incubating anaerobically for $48 \mathrm{~h}$.

4 The same strains were tested for $\alpha$-fucosidase activity, using the chromogenic enzyme substrate 4-nitrophenyl- $\alpha$-L-fucopyranoside (Koch-Light). ${ }^{10}$ The organisms were first grown on Columbia agar, with anaerobic incubation with additional $\mathrm{CO}_{2}$ for five days. A heavy suspension of each test organism was then made in $0.2 \mathrm{ml}$ of a $0.1 \% \mathrm{wt} / \mathrm{vol}$ concentration of the substrate in phosphate buffer, $0.06 \mathrm{~mol} / \mathrm{l}, \mathrm{pH} 8.0$, using a microtitre tray. After $4 \mathrm{~h}$ incubation at $37^{\circ} \mathrm{C}$ the development of a yellow colour, due to release of nitrophenol, indicated $\alpha$-fucosidase activity.

5 Antibiotic sensitivities were determined by the disc agar gel method on blood agar plates incubated anaerobically for $18 \mathrm{~h}$. Discs containing penicillin ( 1 unit) and metronidazole $(1 \mu \mathrm{g})$ were included.

\section{Results}

Following the earlier isolation of seven strains from IUCD wearers, $A$ odontolyticus was isolated from 27 of the 561 IUCD wearers $(4 \cdot 8 \%)$; from four of the 101 patients with pelvic inflammatory disease not known to be IUCD wearers (4\%); and from seven of the 525 vaginal swabs from patients without pelvic inflammatory disease or IUCDs $(1.3 \%)$. Table 1 gives the clinical details of 41 of these patients, together with accompanying bacterial isolates.
In five of the IUCD wearers colonised with $A$ odontolyticus, actinomyces like organisms had been previously reported in cervical smears by Dr AI Spriggs.

The isolates generally conformed to previous descriptions of the organism. ${ }^{11}$ On subculture to horse blood agar, they first appeared as small $\alpha$-haemolytic colonies with surrounding browning of the medium after one to two days' incubation; they later developed a brownish red pigment with a dense centre after three to five days. Pigment production occurred earlier under anaerobic conditions. It was seen only on media containing blood and was more intense in the presence of lysed blood. The pigment was insoluble in water, alcohol, acetone, and ether. Microscopically, the organisms appeared as pleomorphic Gram positive bacteria, showing branching, beaded, or clubbed forms. On subculture, nutrient agar appeared to support growth as well as blood agar. Growth was generally improved in an atmosphere of $5 \% \mathrm{CO}_{2}$ and was maximal under anaerobic conditions with added $\mathrm{CO}_{2}$. Since only prolonged anaerobic incubation was used for primary isolation, it was not established whether any strains were obligate anaerobes initially; but all were facultative on subculture.

Table 2 gives the results of biochemical tests carried out on 28 strains. All were identified unequivocally by the Minitek Anaerobe II System as $A$ odontolyticus. Eleven of the 28 strains showed $\alpha$-fucosidase activity. All strains were sensitive to penicillin and resistant to metronidazole. No strains of $A$ israelii were recognised in any of the cultures included in this study.

\section{Discussion}

Several reports have indicated an association between IUCD use and colonisation of the endocervix by actinomyces like organisms. ${ }^{14-17}$ These were first recognised microscopically in Papanicolaou stained cervical smears from IUCD wearers ${ }^{9}$ and subsequently identified as microcolonies of $A$ israelii by specific immunofluorescence staining. ${ }^{1418}$ In most cases the presence of these "Gupta bodies" is not associated with symptoms, but there is sometimes clinical and pathological evidence of endometritis $^{1718}$ and, rarely, overt actinomycosis of the genital tract may develop. ${ }^{19-21}$

It has been surprisingly difficult to grow actinomyces from the female genital tract, and cultures may be negative even in severe established actinomycosis. ${ }^{1921}$ Strains isolated from cervicovaginal specimens have occasionally been identified as $A$ israelii, ${ }^{14}{ }^{15}$ and $A$ israelii was isolated from the brain abscess of a patient who four years previously 
Table 1 Actinomyces odontolyticus: details of isolations from genital tract specimens

\begin{tabular}{|c|c|c|c|c|c|}
\hline $\begin{array}{l}\text { Case } \\
\text { no }\end{array}$ & Date & Specimen & $\begin{array}{l}\text { IUCD details } \\
\text { where known }\end{array}$ & Clinical details & Associated organism \\
\hline $\begin{array}{l}1 \\
2\end{array}$ & 21. $\begin{array}{r}4.80 \\
4.80\end{array}$ & HVS & $\begin{array}{l}\text { IUCD, } 2 \text { yr } \\
\text { IUCD }\end{array}$ & $\begin{array}{l}\text { Profuse white discharge } \\
\text { No details available }\end{array}$ & \\
\hline 3 & 21. 6.80 & IUCD & IUCD, 5 mo & Abdominal pain & Group C streptococcus \\
\hline 4 & 28. 8.80 & HVS & IUCD & Vaginal discharge & Candida albicans \\
\hline 5 & 30.10 .80 & $\begin{array}{l}\text { Endocervical } \\
\text { swab }\end{array}$ & $\begin{array}{l}\text { Cu ML 250, } \\
6 \mathrm{mo}\end{array}$ & Increasing vaginal discharge & Mixed anaerobes \\
\hline 6 & 8.12 .80 & HVS & IUCD in situ & Vaginal discharge & $\begin{array}{l}\text { GBS, coliforms, Cand } \\
\text { albicans }+++ \text {, bacteroides }\end{array}$ \\
\hline 7 & 10.12 .80 & IUCD & & $\begin{array}{l}\text { Very offensive vaginal } \\
\text { discharge }\end{array}$ & No pathogens \\
\hline 8 & 24. 9.82 & HVS & IUCD & $\begin{array}{l}\text { Irregular periods. Brown } \\
\text { discharge. Painful cervix }\end{array}$ & Peptostreptococcus + \\
\hline 9 & 27. 9.82 & HVS & IUCD & Vaginal discharge & $\begin{array}{l}\text { Mixed anaerobes including } \\
\text { Bacteroides }\end{array}$ \\
\hline 10 & 28. 9.82 & $\begin{array}{l}\text { Endocervical } \\
\text { swab }\end{array}$ & $\begin{array}{l}\text { Saf } \mathrm{T} \text { coil. } \\
4 \mathrm{yr}\end{array}$ & $\begin{array}{l}\text { Tender uterus. Mid-cycle } \\
\text { bleeds. }\end{array}$ & $\begin{array}{l}\text { GBS, coliforms }++, \text { Bacteroides } \\
\text { fragilis }\end{array}$ \\
\hline 11 & 29. 9.82 & HVS & IUCD & $\begin{array}{l}\text { Bloodstained vaginal discharge } \\
\text { for } 6 \text { weeks }\end{array}$ & Peptostreptococcus +++ \\
\hline 12 & 30. 9.82 & HVS & IUCD & Vaginal discharge & $\begin{array}{l}\text { Non-haemolytic streptococcus } \\
\text { faecal streptococcus, } \\
\text { diphtheroids }\end{array}$ \\
\hline 13 & 30.9 .82 & IUCD & Lippes loop & $\begin{array}{l}\text { Removal because of } \\
\text { actinomyces like organisms } \\
\text { in cervical smear }\end{array}$ & Peptococcus, peptostreptococcus \\
\hline 14 & 4.10 .82 & HVS & IUCD & $\begin{array}{l}\text { Abdominal pain? pelvic } \\
\text { inflammatory disease }\end{array}$ & $\begin{array}{l}\text { Cand albicans, non-haemolytic } \\
\text { streptococcus }\end{array}$ \\
\hline \multirow[t]{2}{*}{15} & 11.10 .82 & Endocervical & & & $\begin{array}{l}\text { Group G streptococci pepto- } \\
\text { streptococcus }++\end{array}$ \\
\hline & & IUCD & Lippes loop. & $\begin{array}{l}\text { Actinomyces organisms in } \\
\text { cervical smear }\end{array}$ & Faecal streptococci \\
\hline 16 & 13.10 .82 & HVS & IUCD & $\begin{array}{l}\text { Heavy vaginal discharge } 6 \text { mo } \\
\text { Back pain }\end{array}$ & Bacteroides +++ \\
\hline 17 & 19.10 .82 & $\begin{array}{l}\text { Endocervical } \\
\text { swab }\end{array}$ & IUCD & $\begin{array}{l}\text { Lower abdominal pain } \\
2 \text { weeks }\end{array}$ & No pathogens \\
\hline 18 & 19.10 .82 & HVS & & Vaginal discharge & No pathogens \\
\hline 19 & 19.10 .82 & HVS & & Vaginal discharge & Gardnerella vaginalis +++ \\
\hline 20 & 21.10 .82 & Vaginal swab & & Recurrent vaginal discharge & No pathogens \\
\hline 21 & 25.10 .82 & HVS & IUCD & Recent abdominal pain & $\begin{array}{l}\text { Peptostreptococcus, Cand } \\
\text { albicans, faecal streptococcus }\end{array}$ \\
\hline 22 & 21.10 .82 & IUCD & & $\begin{array}{l}\text { Acute abdominal pain, vaginal } \\
\text { discharge }\end{array}$ & $\begin{array}{l}\text { Streptococci milleri, faecal } \\
\text { streptococcus }\end{array}$ \\
\hline 23 & 24.11 .82 & HVS & IUCD & Vaginal discharge & $\begin{array}{l}\text { Peptostreptococcus Bact } \\
\text { melaninogenicus }\end{array}$ \\
\hline 24 & 1.12 .82 & HVS & & Vaginal discharge, pregnant & No pathogens \\
\hline 25 & 20.12 .82 & HVS & IUCD, 3 yr & $\begin{array}{l}\text { Actinomyces found in routine } \\
\text { cervical smear }\end{array}$ & \\
\hline 26 & 31.12 .82 & IUCD & IUCD & Menorrhagia & Peptostreptococcus \\
\hline 27 & 20.1 .83 & IUCD & CuML 250 & $\begin{array}{l}\text { Actinomyces like organisms } \\
\text { in smear }\end{array}$ & Streptococcus milleri + \\
\hline 28 & 26. 1.83 & $\begin{array}{l}\text { Endocervical } \\
\text { swab }\end{array}$ & $\mathrm{Cu} 7$ & $\begin{array}{l}\text { Pain in left ileac fossa one } \\
\text { month. Pelvic inflammatory } \\
\text { disease. Follicular cystitis }\end{array}$ & Faecal streptococcus, diphtheroids \\
\hline 29 & 31.1 .83 & HVS & IUCD & $\begin{array}{l}\text { Offensive discharge. } \\
\text { ? actinomycosis }\end{array}$ & Faecal streptococcus \\
\hline 30 & 1. 2.83 & HVS & IUCD & Discharge & No pathogens \\
\hline 31 & 4. 2.83 & HVS & IUCD & Discharge & No pathogens \\
\hline 32 & 24. 2.83 & HVS & IUCD & $\begin{array}{l}\text { White vaginal discharge. } \\
\text { Cervicitis }\end{array}$ & GBS \\
\hline 33 & 9. 3.83 & HVS & IUCD & Vaginal discharge $3 \mathrm{mo}$ & $\begin{array}{l}\text { GBS, peptostreptococcus } \\
\text { Gardnerella vaginalis }\end{array}$ \\
\hline 34 & 22. 3.8 .3 & HVS & IUCD & $\begin{array}{l}\text { Actinomycetes seen in cervical } \\
\text { smear. Slight irritant vaginal } \\
\text { discharge with blood staining }\end{array}$ & GBS, Peptostreptococci \\
\hline 35 & 25.3 .83 & IUCD & CuML 250 & & Faecal streptococcus \\
\hline 36 & 26. 5.8 .3 & $\begin{array}{l}\text { Endocervical } \\
\text { swab }\end{array}$ & IUCD & Recent onset of pain & No pathogens \\
\hline 37 & 2. 6.83 & HVS & IUCD, $10 \mathrm{yr}$ & $\begin{array}{l}\text { Offensive vaginal discharge } \\
4 \text { weeks }\end{array}$ & $\begin{array}{l}\text { Peptostreptococcus, faecal } \\
\text { streptococcus }\end{array}$ \\
\hline 38 & 28.6 .83 & HVS & IUCD & Vaginal discharge 3 mo & Peptostreptococcus, diphtheroids \\
\hline 39 & 11.7 .83 & $\begin{array}{l}\text { Endocervical } \\
\text { swab }\end{array}$ & IUCD & Offensive vaginal loss for 2 wk & No pathogens \\
\hline 40 & 21.9 .83 & IUCD & $\begin{array}{l}\text { Dalkon shield, } \\
11 \mathrm{yr}\end{array}$ & Pain and bleeding $3 \mathrm{wk}$ & $\begin{array}{l}\text { Cand albicans, faecal } \\
\text { streptococcus }\end{array}$ \\
\hline 41 & 23. 9.83 & $\begin{array}{l}\text { HVS } \\
\text { IUCD }\end{array}$ & IUCD & $\begin{array}{l}\text { ? Infection. IUCD removed } \\
\text { because of wish to conceive }\end{array}$ & $\begin{array}{l}\text { Cand albicans, faecal } \\
\text { streptococcus, coliforms }\end{array}$ \\
\hline
\end{tabular}

GBS $=$ Group B streptococcus.

IUCD = intrauterine contraceptive device.

HVS $=$ high vaginal swabs

$+=$ scanty $++=$ moderate; $+++=$ profuse growth. 
Table 2 Biochemical characteristics of Actinomyces odontolyticus: results of present study compared with those in previous reports

\begin{tabular}{|c|c|c|c|c|c|c|c|c|c|}
\hline & Batty' & Bergy'" & $\begin{array}{l}\text { Baron } \\
\text { et al }\end{array}$ & $\begin{array}{l}\text { Guillou } \\
\text { et al }^{\circ}\end{array}$ & Holdeman ${ }^{12}$ & $\begin{array}{l}\text { Holmberg } \\
\text { Nord }^{13}\end{array}$ & $\begin{array}{l}\text { Mitchell } \\
\text { et al }\end{array}$ & $\begin{array}{l}\text { Minitek } \\
\text { database }\end{array}$ & $\begin{array}{l}\text { Oxford ser } \\
\text { (28 strains) }\end{array}$ \\
\hline Aesculin hydrolysis & & 63 & - & & + & 49 & + & 80 & 100 \\
\hline $\begin{array}{l}\text { Catalase } \\
\text {. }\end{array}$ & - & 0 & - & - & - & 0 & - & 0 & 0 \\
\hline $\begin{array}{l}\text { Gelatin hydrolysis } \\
\mathrm{H}_{3} \text { S production }\end{array}$ & - & $\begin{array}{c}0 \\
0-87\end{array}$ & & - & - & 0 & - & 0 & 0 \\
\hline $\begin{array}{l}\mathrm{H}_{2} \mathrm{~S} \text { production } \\
\text { Indole production }\end{array}$ & - & $\begin{array}{l}0-87 \\
0\end{array}$ & - & - & _- & 17 & & & \\
\hline Litmus milk-acid & 50 & 80 & - & 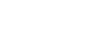 & + & 0 & & 0 & 0 \\
\hline Methyl red & - & 100 & & & & 0 & & & \\
\hline Nitrate reduction & + & 100 & + & + & + & 100 & + & 100 & 100 \\
\hline Starch hydrolysis & & 0 & & - & - & 17 & & 10 & \\
\hline Urease & occas & 0 & - & & - & & & 0 & 0 \\
\hline $\begin{array}{l}\text { Voges-Proskauer } \\
\text { Acid from: }\end{array}$ & & 0 & & & & 0 & & & \\
\hline Adonitol & & 20 & & & - & 0 & & & \\
\hline Arabinose & $\mathrm{v}$ & 50 & - & - & - & 17 & + & 50 & 32 \\
\hline $\begin{array}{l}\text { Cellobiose } \\
\text { Fructose }\end{array}$ & _- & 0 & & + & $\begin{array}{c}- \\
+\end{array}$ & & & 0 & 0 \\
\hline Galactose & $\mathrm{v}$ & & & + & - & 100 & & & \\
\hline Glucose & & 100 & + & + & + & 100 & + & 100 & 100 \\
\hline $\begin{array}{l}\text { Glycerol } \\
\text { Glycogen }\end{array}$ & $\overline{-}$ & $\begin{array}{r}86 \\
0\end{array}$ & - & & $\begin{array}{l}- \\
-\end{array}$ & 0 & & 50 & 50 \\
\hline Lactose & & 77 & - & - & + & 100 & - & 90 & 100 \\
\hline Maltose & - & & + & - & $\mathrm{v}$ & & + & 80 & 100 \\
\hline Mannitol & $\mathrm{v}$ & 0 & - & - & - & 0 & - & 0 & 0 \\
\hline Mannose & & 50 & & - & - & 0 & & 10 & 0 \\
\hline Raffinose & & 50 & & - & - & 0 & & 30 & 46 \\
\hline Rhamnose & - & 43 & & - & - & & & 20 & 0 \\
\hline $\begin{array}{l}\text { Ribose } \\
\text { Salicin }\end{array}$ & & & & & - & 17 & & & \\
\hline $\begin{array}{l}\text { Salicin } \\
\text { Sorbitol }\end{array}$ & - & $\begin{array}{r}70 \\
3\end{array}$ & - & $=$ & $\overline{-}$ & 0 & - & $\begin{array}{r}50 \\
0\end{array}$ & $\begin{array}{r}29 \\
0\end{array}$ \\
\hline Sucrose & $\mathrm{v}$ & & + & + & + & & + & 90 & 100 \\
\hline Trehalose & - & 66 & & - & - & 0 & & 10 & 0 \\
\hline Xylose & - & 53 & - & - & $\mathrm{v}$ & 83 & - & 50 & 25 \\
\hline
\end{tabular}

had developed a tubovarian abscess associated with an IUCD. ${ }^{20}$ In a recent study actinomyces were isolated from 13 of 15 women whose cervical smears showed the presence of actinomyces like bodies. ${ }^{17}$ The cultures were identified as $A$ israelii by immunofluorescence using an antiserum prepared against $A$ israelii which also gave strong reactions with other actinomyces, including $A$ odontolyticus. Since cultures were made on blood agar and there is no mention of the isolation of red colonies, it may be inferred that $\boldsymbol{A}$ odontolyticus was not isolated.

It is uncertain whether actinomyces are indigenous in the normal cervico-vaginal flora; in most studies "Gupta bodies" have been absent from the cervical smears of women not wearing IUCDs. ${ }^{15}{ }^{17}$ In a study using immunofluorescence staining of cervical smears with multiple antisera, $A$ israelii, $A$ naeslundii, and Arachnia propionica were commonly found in women who did not wear IUCDs. ${ }^{22}$ These results were not confirmed in a subsequent study. ${ }^{23}$ In an investigation of patients attending a genitourinary clinic, actinomyces were isolated from the endocervical swabs of 20 of 78 IUCD wearers and from 12 of 58 women using various forms of contraception other than IUCDs. ${ }^{24}$ Twenty nine of the 32 isolates were identified as $A$ israelii by conven- tional biochemical tests. The medium used for isolation in this study did not contain blood, so that red colonies of $\boldsymbol{A}$ odontolyticus would not have been detected. The carbohydrate fermentation reactions of $\boldsymbol{A}$ odontolyticus are variously reported by different authors (Table 2), the results presumably being influenced by the methods used. Identification of actinomyces ultimately depends on analysis of the chemical and antigenic composition of the cell wall, ${ }^{10}$ but such techniques are beyond the scope of a routine laboratory. The $\alpha$-fucosidase reaction appears to be specific for $A$ odontolyticus and $A$ viscosus,$^{10}$ which are readily distinguished by the catalase test. Only 11 of 28 strains in the present study were $\alpha$-fucosidase positive, however, whereas all seven strains tested by Kilian were positive. ${ }^{10}$ The red pigment and aerobic growth of $\boldsymbol{A}$ odontolyticus are important aids to identification; $\boldsymbol{A}$ naeslundii may develop a tan pigment on prolonged incubation but is urease positive.

During 198313 isolations of $A$ odontolyticus from women wearing IUCDs as well as four isolations from non-genital sites were reported to the Communicable Disease Surveillance Centre, Colindale, London, by other laboratories (unpublished observations). It is surprising that previous studies have 
not mentioned $A$ odontolyticus in the female genital tract since it is likely that media containing blood are widely used for cervico-vaginal cultures. Equally, we cannot explain our failure to isolate $A$ israelii in the present study. In five of our patients with $A$ odontolyticus actinomyces like structures had been found previously in cervical smears. It seems likely that these structures may be formed from $A$ odontolyticus in some patients, but this remains to be established, presumably by immunofluorescence staining of cervical smears using specific antibody to $A$ odontolyticus.

The pathogenicity of this organism in the genital tract is unknown, although several of our patients had symptoms which have been attributed to endocervical infection by actinomyces. ${ }^{1617}$ Our findings have not shown unequivocally that IUCD usage predisposes to colonisation by $A$ odontolyticus or that the organism may cause pelvic inflammatory disease in the absence of an IUCD. Further work is required to establish this beyond doubt.

We are grateful to Dr Susan Young for details of isolates of $\boldsymbol{A}$ odontolyticus reported to the Communicable Diseases Surveillance Centre, Colindale.

\section{References}

' Batty I. Actinomyces odontolyticus, a new species of actinomycete regularly isolated from deep carious dentine. J Pathol Bacteriol 1958;75:455-9.

2 Conner HD, Haberman S, Collings CK, Winford TE. Bacteremias following periodontal scaling in patients with healthy appearing gingiva. $J$ Periodont $1967 ; 38: 466-72$.

${ }^{3}$ Morris JF, Kilbourn P. Systemic actinomycosis caused by Actinomyces odontolyticus. Ann Int Med 1974;81:700.

+ Mitchell PD. Hintz CS, Haselby RC. Malar mass due to Actinomyces odontolyticus. J Clin Microbiol 1977;5:658-60.

' Baron EJ, Angevine JM, Sundstrom W. Actinomycotic pulmonary abscess in an immunosuppressed patient. Am J Clin Pathol 1979;72:637-9.

- Guillou J-P, Durieux A. Dublanchet A, Chevrier L. Actinomyces odontolyticus, première étude réalisée en France. $C R$ Acad Sci [D] (Paris) 1977:285: 1561-4.

' Dublanchet A, Durieux R, Guillou JP, Chevrier L, Benclere A.
Un cas de pleurésie purulente á Actinomyces odontolyticus. Medicine et Maladies Infectieuses 1978;8:125-6.

" Raoult D, Kohler JL, Gallais H, Estrangin E, Peloux Y, Casanova P. Septicémie mixte à Fusobacterium necrophorum associé à Actinomyces odontolyticus Pathol Biol (Paris) 1982; 30: 576-80.

' Gupta PK, Hollander DH, Frost JK. Actinomycetes in cervicovaginal smears: an association with IUD usage. Acta Cytol (Baltimore). 1976;20:295.

1" Kilian M. Rapid identification of Actinomycetaceae and related bacteria. J Clin Microbiol 1978;8:127-33.

" Buchanan RE, Gibbons NE, eds: Bergey's manual of determinative bacteriology, 8th ed. Baltimore: Williams and Wilkins, 1974.

12 Holdeman LV, Cato EP, Moore WEC, eds: Anaerobic laboratory manual. Blacksburg VA: Virginia Polytechnic Institute, 1977.

${ }^{13}$ Holmberg K, Nord C-E. Numerical taxonomy and laboratory identification of Actinomyces and Arachnia and some related bacteria. J Gen Microbiol 1975;91:17-44.

14 Bhagavan BS, Gupta PK. Genital actinomycosis and intrauterine contraceptive devices. Cytopathologic diagnosis and clinical significance. Hum Pathol 1978;9:567-78.

is Hager WD, Douglas B, Majmudar B, et al. Pelvic colonisation with Actinomyces in women using intrauterine contraceptive devices. Am J Obstet Gynecol 1979; 135:680-4.

${ }^{16}$ Duguid HLD, Parrat D, Traynor R. Actinomyces-like organisms in cervical smears from women using intrauterine contraceptive devices. Br Med J 1980;281:534-7.

1 Traynor RM, Parrat D, Duguid HLD, Duncan ID. Isolation of actinomycetes from cervical specimens. J Clin Pathol 1981;34:914-6.

Ix Spence MR, Gupta PK, Frost JK, King TM. Cytologic detection and clinical significance of Actinomyces israelii in women using intrauterine contraceptive devices. Am J Obstet Gynecol 1978; 131:295-8.

${ }^{14}$ Charnock M, Chambers TJ. Pelvic actinomycosis and intrauterine contraceptive devices. Lancet 1979;i:1239-40.

${ }^{20}$ de la Monte SM, Gupta PK, White III CL. Systemic Actinomyces infection. A potential complication of intrauterine contraceptive devices. JAMA 1982;248: 1876-7.

2 Gupta PK, Woodruff JD. Actinomyces in vaginal smears. JAMA 1982;247:1175-6.

${ }^{22}$ Pine L, Malcolm GB, Curtis EM, Brown JM. Demonstration of Actinomyces and Arachnia species in cervicovaginal smears by direct staining with species-specific fluorescent-antibody conjugate. J Clin Micrubiol 1981;13:15-21.

${ }^{23}$ Jones JB, Kaplan W, Brown JM, White W. Studies of cervicovaginal smears for the presence of actinomycetes. Mycopathologia 1983;83:53-5.

${ }^{24}$ Grice GC, Hafiz S. Actinomyces in the female genital tract. A preliminary report. Br J Vener Dis 1983;59:317-9. 